\title{
Chronique de la croissance du CIBP
}

\section{Dominique Descotes}

\section{OpenEdition \\ Journals}

Édition électronique

URL : http://journals.openedition.org/ccibp/440

DOI : $10.4000 /$ ccibp.440

ISSN : 2493-7460

\section{Éditeur}

Centre international Blaise Pascal

\section{Édition imprimée}

Date de publication : 15 janvier 1986

Pagination : 1-2

ISSN : 0249-6674

\section{Référence électronique}

Dominique Descotes, "Chronique de la croissance du CIBP », Courrier du Centre international Blaise Pascal [En ligne], 8 | 1986, mis en ligne le 07 décembre 2015, consulté le 24 septembre 2020. URL http://journals.openedition.org/ccibp/440 ; DOI : https://doi.org/10.4000/ccibp.440

Ce document a été généré automatiquement le 24 septembre 2020.

Centre international Blaise Pascal 


\title{
Chronique de la croissance du CIBP
}

\author{
Dominique Descotes
}

1 Notre Courrier $n^{\circ} 7$ rendait essentiellement compte des découvertes du CIBP dans le patrimoine iconographique de l'Auvergne : c'est le rôle du Centre de faire remonter à la lumière les richesses régionales oubliées, défrichant ainsi les « itinéraires pascaliens en Auvergne ». Le numéro 8 au contraire est consacré principalement à la présentation de notre domaine au sein de la Bibliothèque Municipale et Interuniversitaire. Nous proposons à nos correspondants, dans les pages qui suivent, l'édition d'une des propriétés les plus intéressantes du CIBP, la partie pascalienne du manuscrit Usage de la Machine, dont l'acquisition a été annoncée dans notre Courrier $n^{\circ} 4$.

2 Un de nos buts est d'acquérir la documentation pascalienne aussi complètement que possible, afin qu'à longue échéance, Clermont-Ferrand devienne le point de passage obligé de toute recherche approfondie sur Pascal. Quelle que soit la richesse du fonds documentaire de la BMIU, un tel objectif ne peut être atteint du jour au lendemain : le travail d'acquisition est méthodique et lent. De grain en grain, voici où en est notre tas de sable.

3 Nous recevons avec une régularité inégale les publications de nos correspondants. Faut-il battre le rappel ? Un bon signe en revanche : depuis quelques temps, des thèses envoyées par leurs auteurs viennent alimenter notre fonds, qui demeure ainsi à la pointe des recherches pascaliennes.

4 Les achats d'ouvrages se poursuivent, suivant autant que possible les publications nouvelles en matière historique et critique. Quant aux ouvrages anciens, nous avons pu, depuis quelques années, acquérir plusieurs éditions des Pensées et des Provinciales qui nous manquaient, ainsi que quelques ouvrages qui nous ont paru intéressants (les Elementa Geometriae du P. Tacquet par exemple). Mais les livres contemporains qui éclairent la vie intellectuelle de Pascal se trouvent rarement sur le marché, et à des prix souvent excessifs pour nous. Nous faisons donc confectionner systématiquement des microfilms, en commençant par les pièces les plus précieuses du fonds de la BMIU, les Recueils 1036 et 1037, qui contiennent les textes sur la Roulette et sur le vide. Une première vague de commandes a été consacrée aux ouvrages de polémique théologique qui ont servi de sources d'information à Pascal pour la rédaction des Provinciales, des 
Écrits sur la Grâce et des Pensées. Nous avons ainsi acquis les trois Lettres de Bourzeis à un Abbé, à un Président et à un Évêque, la Défense de la Constitution de Lalanne, le Peregrinus Jerichuntinus de Conrius, plusieurs opuscules du P. Desmares, parmi lesquels la Réponse d'un Docteur en Théologie, ainsi que d'autres ouvrages polémiques. Pour l'apologétique, nous disposons, toujours sous forme de microfilms, des Triomphes de la Religion chrétienne du P. Boucher, de L'Impiété des Déistes et de La Vérité des Sciences du P. Mersenne, et surtout du Pugio Fidei de Raymundus Martinus, annoté par Joseph de Voisin. La seconde vague a été consacrée à des textes scientifiques, parmi lesquels figurent d'abord des ouvrages que Pascal a lus ou dont il a connu les auteurs: notre fonds Mersenne s'est enrichi des Préludes de l'Harmonie universelle et des Harmonicorum Libri XII ; nous avons acquis les Cylindricorum et Annularium Libri IV du P. Tacquet, le De Centra Gravitatis de Guldin, les livres de Maurolico, les deux éditions du Mesolabum de Sluse (1659 et 1668), ainsi que les œuvres complètes de Torricelli. Particulièrement intéressants sont aussi les manuscrits mathématiques de Roberval BN 2340 et 9119, qui traitent de la cycloïde, ainsi que l'exemplaire des Lettres de A. Dettonville offert par Pascal à Arnauld et annoté par celui-ci, dont la Bibliothèque de Médecine de Montpellier nous a envoyé la copie. Nous avons procédé de même pour la physique, avec l'acquisition des Essais de Jean Rey par exemple. Parmi nos dernières acquisitions figurent des ouvrages dont le rapport avec Pascal est moins direct, mais qui doivent se montrer utiles pour toute recherche historique: Diophante dans des éditions contemporaines, Bachet de Méziriac, Frenicle de Bessy entre autres. La campagne d'achats va désormais s'orienter vers les grands recueils manuscrits de la tradition pascalienne. Toutes les suggestions de nos correspondants pour l'enrichissement de ce fonds seront les bienvenues.

5 D'autres sources alimentent le fonds du CIBP à la BMIU. Quoiqu'elle ait à présent pris sa retraite de professeur, Thérèse Goyet n'en poursuit pas moins l'exploration des documents de la famille de Keating: plusieurs pièces ont été déposées au Centre sous forme de copies. Nous attendons encore mieux.

6 La recherche iconographique continue (voir le Courrier $\mathrm{n}^{\circ} 7$ ). Nous n'avons pas encore pu obtenir le Blaise Pascal de Rossellini. L'emploi du magnétoscope ouvre quelques perspectives : le CIBP a acquis l'enregistrement d'un film américain dont l'action se déroule dans les milieux de la boxe, et dont un dialogue reprend de très près l'idée du fragment «Infini rien » sur le pari. Là aussi, des découvertes inattendues sont possibles.

7 L'enrichissement $\mathrm{du}$ fonds de CIBP exige de la part de $\mathrm{M}^{\mathrm{me}}$ Durand, notre documentaliste, un gros effort pour le catalogage. Nos visiteurs peuvent à l'heure actuelle disposer de plusieurs fichiers propres à faciliter leurs recherches : un fichier général auteurs, qui comprend tous les ouvrages concernant Pascal et les éditions de ses œuvres, anciens et modernes, localisés à la BMIU ; un fichier du fonds ancien (avant 1810), qui comprend les ouvrages de la BMIU présentant des intérêts divers par rapport à l'œuvre de Pascal (théologiques, philosophiques, scientifiques ou techniques); un fichier bibliographique (livres, articles, contributions dans des périodiques) depuis 1970 ; un fichier matières, bibliographie de première main ( $a u$ vu du document), qui ne comprend donc que les documents traités par le CIBP, indexés selon une liste de descripteurs hiérarchisés, classés par ordre alphabétique; un fichier des microfilms; enfin un fichier de référence intellectuelle, dit "Bibliothèque idéale de Pascal» comprenant les textes que Pascal a lus ou qu'il mentionne. L'informatisation de ces fichiers, qui doit accompagner celle de la Bibliothèque, n'est pas encore commencée ; 
mais la création du «thesaurus » et du fichier matières du CIBP en jette dès à présent les bases.

8 La croissance du CIBP serait impossible sans le soutien qu'il reçoit de ses autorités de tutelle. Nous avons sur ce point une bonne nouvelle à annoncer à nos correspondants : l'Université Clermont II, qui avait fait au Centre une belle place dans la brochure qu'elle a publiée sur ses organismes de recherche, a en plus décidé, sur proposition de son président M. Jacques Fontaine, d'adopter le nom d'Université Blaise Pascal : nous donnons dans ce numéro l'article par lequel a été lancée cette proposition. La Ville de Clermont contribue elle aussi au développement du CIBP, par la récente titularisation de notre documentaliste, $\mathrm{M}^{\mathrm{me}}$ Durand, sur le demi-poste qu'elle occupait jusqu'à présent sur contrat.

9 Malgré tout, un effort est nécessaire pour faire mieux connaître le CIBP à l'extérieur. Nous avons donc produit un dépliant publicitaire dont vous trouverez quelques exemplaires dans le présent envoi. Le dessin de la première page est une composition originale de notre ami Philippe Durin, qui a ressuscité le château de Bien Assis dans nos numéros 3 et 5 . Ce portrait de Pascal saisi en vue de la porte de la demeure des Périer symbolise parfaitement l'esprit de nos recherches sur Blaise Pascal « Auvergnat». Il serait souhaitable que tous nos correspondants contribuent à l'agrandissement du CIBP en assurant la diffusion de ce dépliant à l'échelle amicale. Espérons qu'il éveillera de nombreuses vocations.

Terminons sur les deux manifestations pascaliennes qui se dessinent dans l'avenir. En 1988 se tiendra au Japon un colloque intitulé «Pascal, Port Royal, Orient, Occident ». D'autre part le CIBP projette de renouveler l'expérience de 1976 en proposant un colloque à Clermont sur le thème "Droit et pensée politique autour de Pascal »; les circulaires ne sont pas encore prêtes à partir, mais nous semons l'idée dès maintenant pour qu'elle commence sa germination.

\section{AUTEUR}

DOMINIQUE DESCOTES

CERHAC, Université Blaise Pascal 